\title{
Airport functional damage assessment and prediction based on Bayesian network
}

\author{
Taoju Teng ${ }^{a}$, Qingkun Yu ${ }^{a}$, Feng Wang ${ }^{a}$, Jianwu Wang ${ }^{a}$ \\ Department of Airfield Engineering and Support, Air Force Logistics College, Xuzhou 221000, China \\ a1028419220@qq.com
}

Keywords: airport, damage assessment, Bayesian network.

\begin{abstract}
After the enemy' attack on the airport, how to assess the airport functional damage quickly and scientifically is an important link of the formation of combat effectiveness. This paper is based on the Bayesian theory, and establishes the functional damage and prediction model of airport. With an example of the calculation, the results show that a good simulation response. Under conditions of the regional high-tech war, the airport is the main target of the enemy attack. The damage effect of the airport directly affects the force balance between ourselves and the enemy, so the assessment of airport damage presents the striking study significance. Airport functional damage assessment and prediction refers to a comprehensive description, evaluation, and prediction about the airport system.
\end{abstract}

\section{Bayesian network model overview}

On the base of the Bayesian theorem, Bias network has been developed into directed acyclic graphs which include root nodes, intermediate nodes and leaf nodes, and directed edges connecting nodes. In the graph, each node represents influencing factors, and the relationship between various nodes is determined by the conditional probability function. Bayesian network is characterized by dynamic data updating function, which means if a node changes, other nodes will be influenced, causing the change of the network. Nowadays, Bayesian network model is widely applied in the engineering decision-making, fault diagnosis, analysis and forecast.

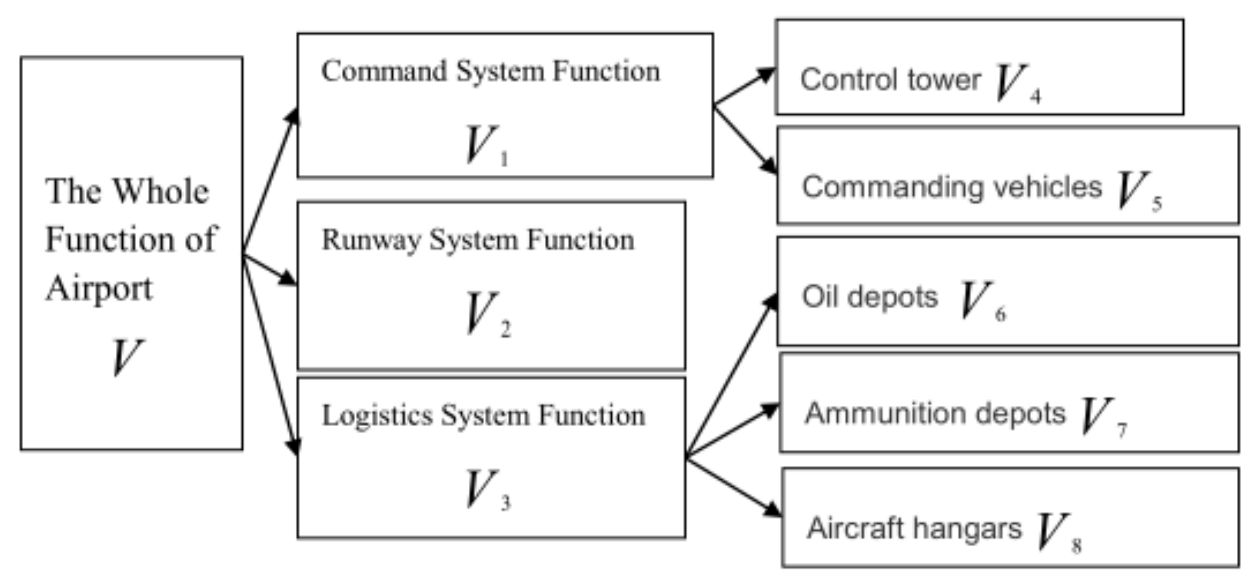

Figure 1 the airport damage assessment model

\section{Bayesian network model for Airport functional damage assessment and prediction}

Taking the main factors affecting the airport functions into consideration, the paper emphasis on logistics system function, airport runway system function and command system function. Airport logistics system consists of oil depots, ammunition depots, aircraft hangars. Airport runway system provides for the platform for taking off and landing, maintenance of aircrafts. The flight control system mainly comprises a fixed control tower and mobile commanding vehicles. According to the 
function analysis and the Bayesian theory, the establishment of the overall function of the airport damage assessment model is shown in figure 1.

Figure 2, $V_{2}, V_{4}, V_{5}, V_{6}, V_{7}, V_{8}$ is the root node, the damage degree of root nodes $p\left(V_{i}\right), p\left(V_{i}\right) \in[0,1], i=4,5,6,7,8, p\left(V_{i}\right)=0$ indicates the weapons miss the target, $p\left(V_{i}\right)=1$ represents the direct hit. According to the Bayesian network theory, the functional damage degree of the whole airport: $R=p(v) \cdot f\left(v\left|v_{1}\right| v_{4}, v_{5}\right) \cdot f\left(v \mid V_{2}\right) \cdot f\left(v\left|V_{3}\right| V_{6}, V_{7}, V_{8}\right)$

In the formula, $f\left(V \mid V_{2}\right)$ means the airport runway system's influence on the function of airport, $f\left(V\left|V_{1}\right| V_{4}, V_{5}\right), f\left(v\left|V_{3}\right| V_{6}, V_{7}, V_{8}\right)$ respectively represents the superposition of impact conditions, the former impacts, $V_{4}$ and $V_{5}$ affect $V_{1}$ at the same time, and then $V_{1}$ influences $V$.The latter $V_{6}, V_{7}, V_{8}$ simultaneously affects $V_{3}$, and then $V_{3}$ influences $V$.Thus, the formula (1) is the damage assessment model for airport function based on Bayesian network after the assessment model is established, the conditional probability function like $f\left(v \mid v_{2}\right)$ is quantified. It's difficult to quantify the conditional probability function. This paper solves the problem in a scientific method combined with qualitative and quantitative analysis.

(1) Runway system function. With more craters are scattered in the runway, the whole function of the airport is worse; on the contrary, the craters are less, the whole function of the airport is better. Assuming that the impact on the overall function of the airport is a linear function $f\left(v \mid v_{2}\right)=a_{1} \cdot v_{1}+a_{2}, a_{1}, a_{2}$ are the undetermined coefficients. $v_{2}=0$, the value of the conditional probability function is $0 ; v_{2}=1$, the value of the conditional probability function is 0.95. Under these conditions, we can obtain the value of $\boldsymbol{a}_{1}$ and $\boldsymbol{a}_{2}, \boldsymbol{a}_{1}=-0.05, \boldsymbol{a}_{2}=1$. So the conditional probability function is $f\left(v \mid v_{2}\right)=1-0.05 \mathrm{v}$.

(2) Command system function and logistics system function.Similarly, we can calculate the value of the conditional probability function of each factor of command system function and logistics system function, as shown in table 1.

Table 1 The conditional probability function of command system function and logistics system function

$$
\begin{array}{ll}
f\left(v \mid V_{1}\right)=0.4 \cdot v_{1}+0.6 & f\left(v_{1} \mid v_{4}\right)=-0.94 \cdot v_{4}^{2}+1.94 \cdot v_{4}+0 \\
& f\left(v_{1} \mid v_{5}\right)=-0.63 v_{5}^{2}+1.63 v_{5}
\end{array}
$$$$
f\left(V \mid V_{3}\right)=e^{0.53}-0.7
$$

$$
\begin{aligned}
& f\left(v_{3} \mid v_{6}\right)=0.5 v_{6}+0.5 \\
& f\left(v_{3} \mid v_{7}\right)=0,55 v_{7}+0.45 \\
& f\left(v_{3} \mid v_{8}\right)=0.45 v_{8}+0.55
\end{aligned}
$$

\section{Example analysis}

Assuming that the airport suffered from the attack, our personnel collect some relevant information on the damage of airport, the values of root nodes in the Bayesian network are estimated, as shown in table 2 
In the next, the values of root nodes are substituted the conditional probability function. Other nodes' values are shown in table 3.

Table 2 The expert' assessment of root nodes' values

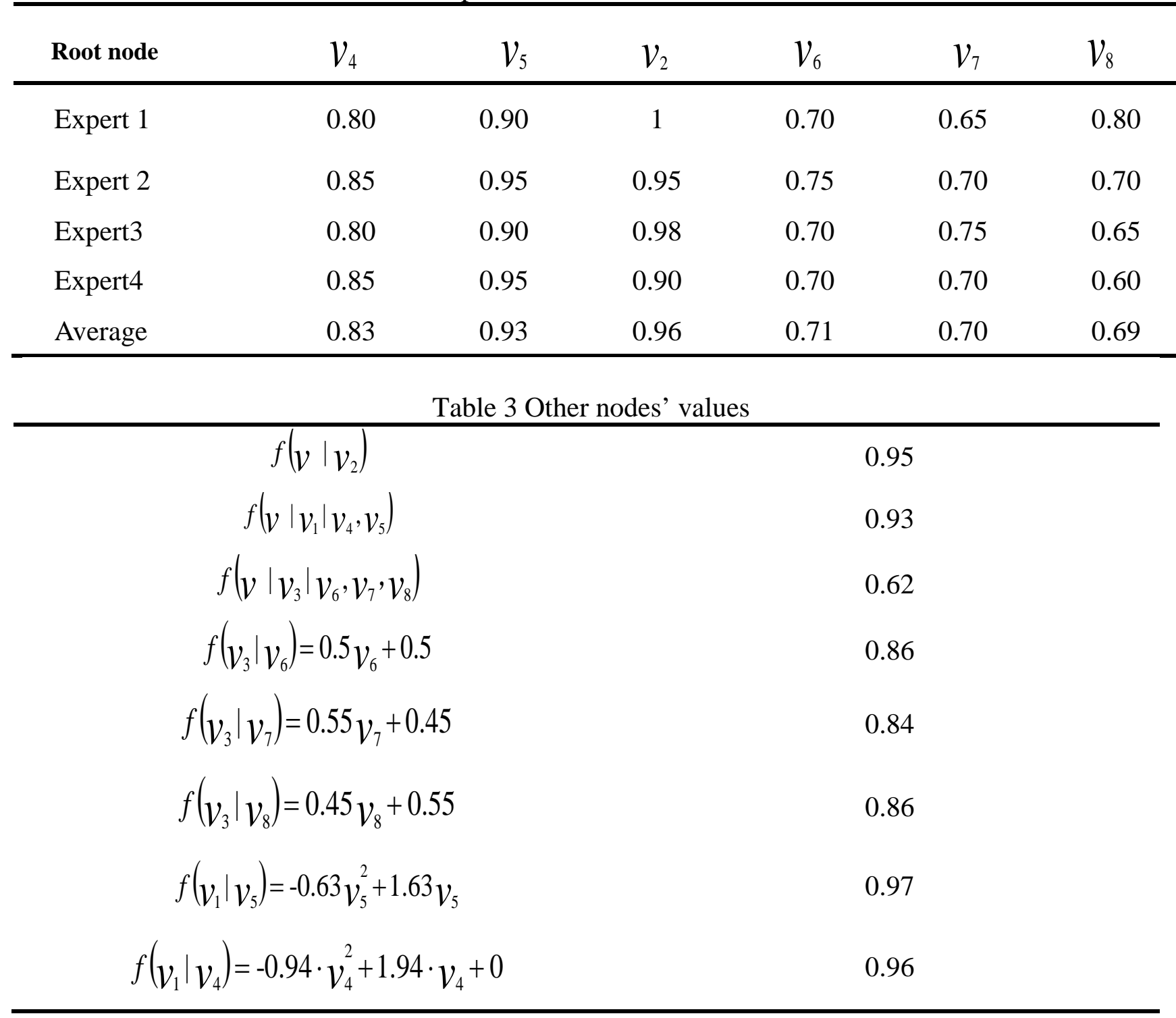

In ideal conditions, the degree of the airport damage is as the following.

$R=1 \cdot 0.95 \cdot 0.93 \cdot 0.62=0.55$

It can be inferred that the level of the airport damage is medium injury.

After the attack on the airport, the damage information collected by expert's shows that craters are uniformly distributed on the runway, the oil depot has a serious injury, and aircraft hangars are slightly damaged. The assessment of results grades medium injury.

\section{Conclusion}

In the process of airport functional damage assessment and prediction, Bayesian network is to deal with the uncertainty arising from different compositions, and provides visual diagrams model. This paper conducted a preliminary study on the application of Bayesian network to evaluate airport functional damage, through the calculation example. The model assessment values are similar with the actual situation. 


\section{Reference}

[1] Yang Feng, Wang Weiping. Simulation and evaluation of weapon equipments [M]. Beijing: Publishing House of electronics industry, 2010.

[2] Yu Lei. Missile defense' effectiveness study based on Bayesian network model. [J] Xi'an: Northwestern Polytechnical University, 2012.

[3] Luo Laike, Chen Jun. Effectiveness Analysis of Fire Fight Based on SEA Method [J].Ordnance Industry Automation, 2009. 